\title{
NON-UNIFORM CONTINUITY OF THE FLOW MAP FOR AN EVOLUTION EQUATION MODELING SHALLOW WATER WAVES OF MODERATE AMPLITUDE
}

\author{
NILAY DURUK MUTLUBAŞ, ANNA GEYER, AND BOGDAN-VASILE MATIOC
}

\begin{abstract}
We prove that the flow map associated to a model equation for surface waves of moderate amplitude in shallow water is not uniformly continuous in the Sobolev space $H^{s}$ with $s>3 / 2$. The main idea is to consider two suitable sequences of smooth initial data whose difference converges to zero in $H^{s}$, but such that neither of them is convergent. Our main theorem shows that the exact solutions corresponding to these sequences of data are uniformly bounded in $H^{s}$ on a uniform existence interval, but the difference of the two solution sequences is bounded away from zero in $H^{s}$ at any positive time in this interval. The result is obtained by approximating the solutions corresponding to these initial data by explicit formulae and by estimating the approximation error in suitable Sobolev norms.
\end{abstract}

\section{IntRoduCtion AND THE MAIN RESUlT}

We consider a model equation for surface waves of moderate amplitude in shallow water

$$
u_{t}+u_{x}+6 u u_{x}-6 u^{2} u_{x}+12 u^{3} u_{x}+u_{x x x}-u_{x x t}+14 u u_{x x x}+28 u_{x} u_{x x}=0
$$

which arises as an approximation of the Euler equations in the context of homogenous, inviscid gravity water waves. In recent years, several nonlinear models have been proposed in order to understand some important aspects of water waves, like wave breaking or solitary waves. One of the most prominent examples is the Camassa-Holm $(\mathrm{CH})$ equation [3], which is an integrable, infinite-dimensional Hamiltonian system $[1,4,7]$. The relevance of the $\mathrm{CH}$ equation as a model for the propagation of shallow water waves was discussed by Johnson [19], where it is shown that it describes the horizontal component of the velocity field at a certain depth within the fluid; see also [5]. Building upon the ideas presented in [19], Constantin and Lannes [8] have recently derived the evolution equation (1) as a model for the motion of the free surface of the wave, and they evince that (1) approximates the governing equations to the same order as the $\mathrm{CH}$ equation. Besides deriving (1), the authors of [8] also establish the local well-posedness results for the Cauchy problem associated to (1). Relying on a semigroup approach due to Kato [21], Duruk [10] has shown that this feature holds for a larger class of initial data, as well as for solutions which are spatially periodic [11]. The well-posedness in the context of Besov spaces together with the regularity and the persistance properties of strong solutions are studied in [26].

Similarly to the $\mathrm{CH}$ equation, cf. [6, 25], the model equation (1) can also capture the phenomenon of wave breaking: for certain initial data the solution remains bounded, but its slope becomes unbounded in finite time cf. [8, 11]. Unlike for the $\mathrm{CH}$ equation, which is known to posses global solutions, cf. [2, 6], it is not apparent how to control the solutions of (1) globally, due to the fact that this equation involves higher order nonlinearities in $u$

Date: December 11, 2013.

2010 Mathematics Subject Classification. 35B30, 35G25, 35L05 .

Key words and phrases. Camassa-Holm equation; flow map; non-uniform continuity; water waves. 
and its derivatives than the $\mathrm{CH}$ equation. On the other hand, if one passes to a moving frame, it can be shown that there exist solitary travelling wave solutions decaying at infinity [14]. Their orbital stability has been recently studied in [9] using an approach proposed by Grillakis, Shatah and Strauss [15], which takes advantage of the Hamiltonian structure of (1).

In the present paper, we consider the Cauchy problem associated to (1) in the setting of periodic functions. From the local well-posedness results [11, 10], we know that its solutions depend continuously on their corresponding initial data in Sobolev spaces $H^{s}$ with $s>3 / 2$. Our main result states that this dependence is not uniformly continuous. This property was only recently shown to hold true for the $\mathrm{CH}$ equation [16, 17], and was subsequently confirmed also for the Euler equations [18] and for several related hyperbolic problems such as the $\mu-b$ equation [23], the hyperelastic rod equation [20], for a modified $\mathrm{CH}$ system [24], and for the modified $\mathrm{CH}$ equation [13]. The main difficulty we encounter compared to all these references is that, as mentioned before, our equation has a higher degree of nonlinearity. Nevertheless, we were able two find two sequences of smooth initial data whose difference converges to zero in $H^{s}$, but such that none of them is convergent, with the corresponding solutions of (1) being uniformly bounded on a common (nonempty) interval of existence. Approximating these solutions by explicit formulae, we then successively estimate the error in suitable Sobolev norms and use well-known interpolation properties of the Sobolev spaces and commutator estimates to show that at any time of the common existence interval the difference of the two sequences of exact solutions is bounded from below in the $H^{s}$-norm by a positive constant. More precisely, denoting by $u\left(\cdot ; u_{0}\right)$, the unique solution of (1) corresponding to the initial data $u_{0} \in H^{s}(\mathbb{S})$ with $s>3 / 2$, cf. Theorem 2.1 , our main result states:

Theorem 1.1 (Non-uniform continuity of the flow map). For $s>3 / 2$, the flow map

$$
u_{0} \mapsto u\left(\cdot ; u_{0}\right): H^{s}(\mathbb{S}) \rightarrow C\left([0, T), H^{s}(\mathbb{S})\right) \cap C^{1}\left([0, T), H^{s-1}(\mathbb{S})\right)
$$

associated to the evolution equation (1) is continuous, but it is not uniformly continuous. More precisely, there exist two sequences of solutions

$$
\left(u_{n}\right)_{n},\left(\widetilde{u}_{n}\right)_{n} \subset C\left(\left[0, T_{u}\right], H^{s}(\mathbb{S})\right) \cap C^{1}\left(\left[0, T_{u}\right], H^{s-1}(\mathbb{S})\right),
$$

where $T_{u}>0$, and a positive constant $C>0$ with the following properties:

$$
\begin{aligned}
& \sup _{n \in \mathbb{N}} \max _{\left[0, T_{u}\right]}\left\|u_{n}\right\|_{H^{s}}+\left\|\widetilde{u}_{n}\right\|_{H^{s}} \leq C, \\
& \lim _{n \rightarrow \infty}\left\|u_{n}(0)-\widetilde{u}_{n}(0)\right\|_{H^{s}}=0,
\end{aligned}
$$

but

$$
\liminf _{n \rightarrow \infty}\left\|u_{n}(t)-\widetilde{u}_{n}(t)\right\|_{H^{s}} \geq C^{-1}|\sin (t)| \quad \text { for } t \in\left(0, T_{u}\right] .
$$

The structure of the paper is as follows: In Theorem 2.1 we recall some properties concerning the well-posedness of (1) from [11] and determine a lower bound on the existence time of the solution in $H^{s}$ in terms of the initial data. Then, we introduce two sequences of approximate solutions $\left(u^{\omega, n}\right)_{n}, \omega \in\{-1,1\}$, and compute the approximation error in Lemma 3.1. The corresponding solutions $u_{\omega, n}$ of (1) determined by the initial data $u^{\omega, n}(0)$ are then shown to be uniformly bounded on a common interval of existence, the absolute error $\left\|u^{\omega, n}-u_{\omega, n}\right\|$ being computed in different Sobolev norms, cf. Lemmas 4.1-4.3. We end the paper with the proof of the main result. 
Notation. Throughout this paper, we shall denote by $C$ positive constants which may depend only upon $s$. Furthermore, $H^{r}:=H^{r}(\mathbb{S})$, with $r \in \mathbb{R}$, is the $L_{2}$-based Sobolev space on the circle $\mathbb{S}:=\mathbb{R} / \mathbb{Z}$. Given $r \in \mathbb{R}$, we let $\Lambda^{r}:=\left(1-\partial_{x}^{2}\right)^{r / 2}$ denote the Fourier multiplier with symbol $\left(\left(1+|k|^{2}\right)^{r / 2}\right)_{k \in \mathbb{Z}}$. It is well-known that $\Lambda^{r}: H^{q}(\mathbb{S}) \rightarrow H^{q-r}(\mathbb{S})$ is an isometric isomorphism for all $q, r \in \mathbb{R}$. Furthermore, the Banach space $W_{\infty}^{m}:=W_{\infty}^{m}(\mathbb{S})$, $m \in \mathbb{N}$, consisting of all bounded functions which possess bounded weak derivatives of order less than or equal to $n$, is endowed with the usual norm.

Some useful estimates. The following commutator estimates play a crucial role in our analysis:

$$
\begin{aligned}
& \left\|\left[\Lambda^{r}, f\right] g\right\|_{L_{2}} \leq C_{r}\left(\left\|f_{x}\right\|_{L_{\infty}}\left\|\Lambda^{r-1} g\right\|_{L_{2}}+\left\|\Lambda^{r} f\right\|_{L_{2}}\|g\|_{L_{\infty}}\right) \quad \text { for all } r>3 / 2 \\
& \left\|\left[\Lambda^{\sigma} \partial_{x}, f\right] g\right\|_{L_{2}} \leq C\|f\|_{H^{s}}\|g\|_{H^{\sigma}} \quad \text { for } s>3 / 2 \text { and } 1+\sigma \in[0, s] .
\end{aligned}
$$

They hold for all functions $f, g \in C^{\infty}(\mathbb{S})$ and for the commutator $[S, T]:=S T-T S$. The Calderon-Coifman-Meyer estimate (3) follows from Proposition 4.2 in Taylor [30]. The estimate (2) is due to Kato and Ponce [22, 29]. Additionally, we shall use the following multiplier inequality

$$
\|f g\|_{H^{t}} \leq C\|f\|_{H^{t}}\|g\|_{H^{r}} \quad \text { for } t \leq r, r>1 / 2
$$

and $f \in H^{t}(\mathbb{S}), g \in H^{r}(\mathbb{S})$, cf. e.g. [28].

\section{The LOCAL WELL-POSEDNESS RESUlT}

Using the above notation, we observe that the evolution problem associated to (1) can be rendered as the following Cauchy problem:

$$
\left\{\begin{aligned}
u_{t} & =u_{x}+14 u u_{x}+\partial_{x} \Lambda^{-2} R \quad \text { for } t>0 \\
u(0) & =u_{0}
\end{aligned}\right.
$$

where $R:=R(u)$ is defined as

$$
R(u):=7 u_{x}^{2}-3 u^{4}+2 u^{3}-10 u^{2}-2 u .
$$

Relying upon the local well-posedness results established in [11] for the quasilinear Cauchy problem (5), we determine in the following theorem a lower bound for the maximal existence time of the solutions in terms of Sobolev norms of the initial data. Additionally, we obtain a bound on the $H^{s}$-norm of the local strong solutions on this particular existence interval.

Theorem 2.1. Let $s>3 / 2$ be given. Then, we have:

(i) The problem $(5)$ possesses for each $u_{0} \in H^{s}(\mathbb{S})$ a unique maximal solution

$$
u\left(\cdot ; u_{0}\right) \in C\left([0, T), H^{s}(\mathbb{S})\right) \cap C^{1}\left([0, T), H^{s-1}(\mathbb{S})\right),
$$

whereby $T=T\left(u_{0}\right)$. Moreover, the flow map

$$
u_{0} \mapsto u\left(\cdot ; u_{0}\right): H^{s}(\mathbb{S}) \rightarrow C\left([0, T), H^{s}(\mathbb{S})\right) \cap C^{1}\left([0, T), H^{s-1}(\mathbb{S})\right)
$$

is continuous.

(ii) Given $u_{0} \in H^{s}(\mathbb{S})$, the maximal existence time of the solution $u\left(\cdot ; u_{0}\right)$ of (5) satisfies

$$
T>T_{0}:=\frac{\left\|u_{0}\right\|_{H^{1}}^{5}}{2 C\left(1+\left\|u_{0}\right\|_{H^{1}}^{5}\right)\left\|u_{0}\right\|_{H^{s}}^{3}}
$$

where $C$ is a positive constant.

(iii) We have

$$
\|u(t)\|_{H^{s}} \leq 2\left\|u_{0}\right\|_{H^{s}} \quad \text { for all } t \in\left[0, T_{0}\right] .
$$


Before proceeding with the proof, one can show by using integration by parts shows $[8,11]$ that the $H^{1}$-norm of the solutions of (1) is preserved in time when $s \geq 2$. Based upon this observation and relying on Theorem $2.1(i)$, we then find that

$$
\|u(t)\|_{H^{1}}=\left\|u_{0}\right\|_{H^{1}} \quad \text { for all } u_{0} \in H^{s}(\mathbb{S}), s>3 / 2 \text {, and } 0 \leq t<T .
$$

Proof of Theorem 2.1. The assertion (i) follows from the local well-posedness results established in [11]. For $(i i)$, we first pick $u_{0} \in H^{s}(\mathbb{S})$ with $u_{0} \neq 0$ and denote by $T$ the maximal existence time of the associated solution $u=u\left(\cdot ; u_{0}\right)$. In order to determine a lower bound for $T$, we first show that $\|u\|_{H^{s}}^{2}$ satisfies a differential inequality. We proceed as in $[27,29]$ and pick a Friedrichs mollifier ${ }^{1} \mathcal{J}_{\varepsilon} \in O P S^{-\infty}, \varepsilon \in(0,1)$. Since $\mathcal{J}_{\varepsilon}$ is itself a Fourier multiplier, the time evolution of the $H^{s}$-norm of $\mathcal{J}_{\varepsilon} u$ is given by

$$
\frac{1}{2} \frac{d}{d t}\left\|\mathcal{J}_{\varepsilon} u\right\|_{H^{s}}^{2}=\frac{1}{2} \frac{d}{d t}\left\|\Lambda^{s} \mathcal{J}_{\varepsilon} u\right\|_{L_{2}}^{2}=\int_{\mathbb{S}} \Lambda^{s} \mathcal{J}_{\varepsilon} u \Lambda^{s} \mathcal{J}_{\varepsilon} u_{t} d x=I_{1}+I_{2}
$$

where

$$
I_{1}:=\int_{\mathbb{S}} \Lambda^{s} \mathcal{J}_{\varepsilon} u \Lambda^{s} \mathcal{J}_{\varepsilon}\left(u u_{x}\right) d x, \quad I_{2}:=\int_{\mathbb{S}} \Lambda^{s} \mathcal{J}_{\varepsilon} u \Lambda^{s} \mathcal{J}_{\varepsilon}\left(\partial_{x} \Lambda^{-2} R\right) d x .
$$

The latter equality is based on the observation that

$$
\int_{\mathbb{S}} \Lambda^{s} \mathcal{J}_{\varepsilon} u \Lambda^{s} \mathcal{J}_{\varepsilon} u_{x} d x=\int_{\mathbb{S}} \Lambda^{s} \mathcal{J}_{\varepsilon} u \partial_{x}\left(\Lambda^{s} \mathcal{J}_{\varepsilon} u\right) d x=0
$$

To estimate the first term, we use the following bound which was derived in Taylor [29], by means of the Kato-Ponce estimate (2):

$$
\left|I_{1}\right| \leq C\|u\|_{W_{\infty}^{1}}\|u\|_{H^{s}}^{2} .
$$

Employing the Cauchy-Schwartz inequality and the algebra property of $H^{r}(\mathbb{S}), r>1 / 2$, the term $I_{2}$ can be estimated as follows:

$$
\left|I_{2}\right| \leq\left\|\mathcal{J}_{\varepsilon} \Lambda^{s} u\right\|_{L_{2}}\left\|\mathcal{J}_{\varepsilon} \Lambda^{s}\left(\partial_{x} \Lambda^{-2} R\right)\right\|_{L_{2}} \leq C\|u\|_{H^{s}}\|R\|_{H^{s-1}} \leq C\left(1+\|u\|_{H^{s}}^{5}\right) .
$$

Finally, we combine these estimates and let $\varepsilon$ tend to 0 to find that

$$
\frac{d}{d t}\|u\|_{H^{s}}^{2} \leq C\left(1+\|u\|_{H^{s}}^{5}\right) \quad \text { for all } t \in[0, T) .
$$

Recalling that the $H^{1}$-norm of $u$ is preserved in time, we get $\left\|u_{0}\right\|_{H^{1}} \leq\|u(t)\|_{H^{s}}$ for all $t \in[0, T)$, and together with (9) we find that

$$
\frac{d}{d t}\|u\|_{H^{s}}^{2} \leq C \frac{1+\left\|u_{0}\right\|_{H^{1}}^{5}}{\left\|u_{0}\right\|_{H^{1}}^{5}}\|u\|_{H^{s}}^{5} \quad \text { for all } t \in[0, T) .
$$

We conclude that

$$
\|u(t)\|_{H^{s}} \leq \frac{\left\|u_{0}\right\|_{H^{s}}}{\left(1-C \frac{1+\left\|u_{0}\right\|_{H^{1}}^{5}}{\left\|u_{0}\right\|_{H^{1}}^{5}}\left\|u_{0}\right\|_{H^{s}}^{3} t\right)^{1 / 3}} \quad \text { for all } t<\max \left\{\frac{\left\|u_{0}\right\|_{H^{1}}^{5}}{C\left(1+\left\|u_{0}\right\|_{H^{1}}^{5}\right)\left\|u_{0}\right\|_{H^{s}}^{3}}, T\right\} .
$$

It follows that the constant $T_{0}$ defined by the relation (7) is a lower bound for $T$, and that $\|u(t)\|_{H^{s}} \leq 2\left\|u_{0}\right\|_{H^{s}}$ for all $t \leq T_{0}$. This proves the claim.

\footnotetext{
${ }^{1}$ Choosing $\rho \in C_{0}^{\infty}(\mathbb{R})$ with supp $\rho \subset(-1 / 2,1 / 2)$ and setting $\rho_{\varepsilon}(x):=\varepsilon^{-1} \rho(x / \varepsilon)$ for $\varepsilon \in(0,1)$ and $x \in \mathbb{R}$, the mollifier $\mathcal{J}_{\varepsilon}$ is defined by $\mathcal{J}_{\varepsilon} u:=\rho_{\varepsilon} * u$ for all $u \in L_{2}(\mathbb{S})$. For every $\varepsilon \in(0,1)$ and $s>0$, we have that $\mathcal{J}_{\varepsilon}: L_{2}(\mathbb{S}) \rightarrow H^{k}(\mathbb{S})$ is continuous, $\left\|\mathcal{J}_{\varepsilon} u-u\right\|_{H^{s}} \rightarrow_{\varepsilon \rightarrow 0} 0$ for all $u \in H^{s}(\mathbb{S})$, and $\mathcal{J}_{\varepsilon}: L_{2}(\mathbb{S}) \rightarrow L_{2}(\mathbb{S})$ is a contraction. Being a Fourier multiplier, $\mathcal{J}_{\varepsilon}$ commutes with $\partial_{t}, \partial_{x}$, and $\Lambda^{s}, s>0$, cf. e.g [12].
} 


\section{Approximate solutions for the EVOlution EQUATION}

In the following we consider approximate solutions of the evolution equation (1) of the form

$$
u^{\omega, n}(t, x):=\frac{\omega n^{-1}-1-n^{-s} \cos (n x+\omega t)}{14},
$$

where $\omega \in\{-1,1\}$ and $n \in \mathbb{N} \backslash\{0\}$. When $n$ is very large, the term involving the cosine has a high spatial frequency whereas the other term is constant. Before we estimate the error of these approximate solutions, observe that

$$
\|\sin (n x-\alpha)\|_{H^{\sigma}}=\|\cos (n x-\alpha)\|_{H^{\sigma}}=\sqrt{\pi}\left(1+n^{2}\right)^{\sigma / 2}
$$

for all $\alpha, \sigma \in \mathbb{R}$ and $n \in \mathbb{N} \backslash\{0\}$. Indeed, the functions $\phi_{n}:=e^{i n \cdot} / \sqrt{2 \pi}, n \in \mathbb{Z}$, form an orthonormal basis of $L_{2}(\mathbb{S})$, and therefore a direct computation (see also [17, Lemma 1]) shows that

$$
\begin{aligned}
\|\cos (n x-\alpha)\|_{H^{\sigma}}^{2} & =\frac{\left(1+n^{2}\right)^{\sigma}}{2 \pi}\left(\left|\int_{0}^{2 \pi} \cos (n x-\alpha) e^{-i n x} d x\right|^{2}+\left|\int_{0}^{2 \pi} \cos (n x-\alpha) e^{i n x} d x\right|^{2}\right) \\
& =\frac{\left(1+n^{2}\right)^{\sigma}}{2 \pi}\left(\left|\pi e^{-i \alpha}\right|^{2}+\left|\pi e^{i \alpha}\right|^{2}\right)=\pi\left(1+n^{2}\right)^{\sigma} .
\end{aligned}
$$

We emphasize that in contrast to [17], due to additional terms appearing in (1) the precise computation of (12) is very important when estimating the norm of $u^{\omega, n}$ in $H^{s}(\mathbb{S})$. In view of (12) and noting that $\|1\|_{H^{\sigma}}=\sqrt{2 \pi}$, we obtain the bound

$$
\left\|u^{\omega, n}\right\|_{H^{\sigma}} \leq C\left(1+n^{\sigma-s}\right) \quad \text { for all } \sigma>0, \omega \in\{-1,1\} \text {, and } n \in \mathbb{N} \backslash\{0\} .
$$

Substituting the approximate solution $u^{\omega, n}$ into the equation (1) the following expression for the error is found:

$$
E:=u_{t}^{\omega, n}-u_{x}^{\omega, n}-14 u^{\omega, n} u_{x}^{\omega, n}-\partial_{x} \Lambda^{-2} R\left(u^{\omega, n}\right)
$$

Lemma 3.1 (Estimating the error of approximate solutions). Given $s>3 / 2$, there is a positive constant $C$ such that

$$
\|E\|_{H^{\sigma}} \leq C \begin{cases}n^{-2 s+1+\sigma}, & \text { if } 3 / 2<s<2, \\ n^{-s-1+\sigma}, & \text { if } s \geq 2,\end{cases}
$$

for all $1 / 2<\sigma \leq 1, \omega \in\{-1,1\}$, and $n \in \mathbb{N} \backslash\{0\}$.

Proof. Observe that $E=E_{1}-E_{2}$, where

$$
\begin{aligned}
& E_{1}:=u_{t}^{\omega, n}-u_{x}^{\omega, n}-14 u^{\omega, n} u_{x}^{\omega, n}=\frac{n^{-2 s+1}}{28} \sin (2(n x+\omega t)), \\
& E_{2}:=\Lambda^{-2}\left(14 u_{x}^{\omega, n} u_{x x}^{\omega, n}-12\left(u^{\omega, n}\right)^{3} u_{x}^{\omega, n}+6\left(u^{\omega, n}\right)^{2} u_{x}^{\omega, n}-20 u^{\omega, n} u_{x}-2 u_{x}^{\omega, n}\right) .
\end{aligned}
$$

Recalling (12) and the fact that $\left\|u^{\omega, n}\right\|_{H^{1}} \leq C$ for all $n \geq 1, \omega \in\{-1,1\}$, cf. (13), we obtain that

$$
\begin{aligned}
\left\|E_{1}\right\|_{H^{\sigma}} \leq C n^{-2 s+1+\sigma} & \\
\left\|E_{2}\right\|_{H^{\sigma}} \leq & C\left(\left\|u_{x}^{\omega, n} u_{x x}^{\omega, n}\right\|_{H^{\sigma-2}}+\left\|\left(u^{\omega, n}\right)^{3} u_{x}^{\omega, n}\right\|_{H^{\sigma-2}}+\left\|\left(u^{\omega, n}\right)^{2} u_{x}^{\omega, n}\right\|_{H^{\sigma-2}}\right. \\
& \left.\quad+\left\|u^{\omega, n} u_{x}^{\omega, n}\right\|_{H^{\sigma-2}}+\left\|u_{x}^{\omega, n}\right\|_{H^{\sigma-2}}\right) \\
\leq & {\left[n^{-2 s+3}\|\sin (2(n x+\omega t))\|_{H^{\sigma-2}}\right.} \\
& \left.\quad+\left(\left\|u^{\omega, n}\right\|_{H^{1}}^{3}+\left\|u^{\omega, n}\right\|_{H^{1}}^{2}+\left\|u^{\omega, n}\right\|_{H^{1}}+1\right)\left\|u_{x}^{\omega, n}\right\|_{H^{\sigma-2}}\right] \\
\leq & C\left(n^{-2 s+1+\sigma}+n^{-s-1+\sigma}\right),
\end{aligned}
$$


where we have employed the multiplier inequality (4). Combining (16) and (17) we obtain the desired conclusion.

\section{ERror estimates}

For each $\omega \in\{-1,1\}$ and $n \in \mathbb{N} \backslash\{0\}$, we consider the solution $u_{\omega, n}$ of equation (1) whose initial data is given by the approximate solution $u^{\omega, n}$ evaluated at time zero, i.e. $u_{\omega, n}$ satisfies the equations

$$
\left\{\begin{aligned}
\partial_{t} u_{\omega, n} & =u_{\omega, n} \partial_{x} u_{\omega, n}+14 u_{\omega, n} \partial_{x} u_{\omega, n}+\partial_{x} \Lambda^{-2} R\left(u_{\omega, n}\right) \quad t>0 \\
u_{\omega, n}(0) & =u^{\omega, n}(0)
\end{aligned}\right.
$$

Note that $u_{\omega, n}(0)$ is bounded in $H^{s}(\mathbb{S})$ for any $s \in \mathbb{R}$. Indeed, since $u_{\omega, n}(0)=u^{\omega, n}(0)$ and recalling the definition (11) we find that

$$
\left|\left\|u_{\omega, n}(0)\right\|_{H^{s}}-\frac{\|1\|_{H^{s}}}{14}\right| \leq \frac{\left\|\omega n^{-1}\right\|_{H^{s}}+n^{-s}\|\cos (n x)\|_{H^{s}}}{14}
$$

which yields

$$
\frac{\sqrt{\pi}}{28} \leq \liminf _{n \rightarrow \infty}\left\|u_{\omega, n}(0)\right\|_{H^{s}} \leq \limsup _{n \rightarrow \infty}\left\|u_{\omega, n}(0)\right\|_{H^{s}} \leq \frac{\sqrt{2 \pi}}{7}
$$

for $\omega \in\{-1,1\}$. Furthermore, we obtain in view of $(12)$ that

$$
\lim _{n \rightarrow \infty}\left\|u_{\omega, n}(0)\right\|_{H^{1}}=\frac{\sqrt{2 \pi}}{14}
$$

for $\omega \in\{-1,1\}$. Therefore, if $s>3 / 2$ we may infer from the Theorem 2.1 that there exists an integer $n_{0} \geq 1$ and positive constants $C$ and $T_{u} \leq T_{0}\left(u_{\omega, n}(0)\right)$, such that

$$
\left\|u_{\omega, n}(t)\right\|_{H^{s}} \leq C
$$

for all $t \in\left[0, T_{u}\right], n \geq n_{0}$ and $\omega \in\{-1,1\}$. In the following lemma, we find that the exact solutions $u_{\omega, n}$ have very nice regularity properties, which allow us to estimate the difference to the approximate solution $u^{\omega, n}$ as follows:

Lemma 4.1 (Estimating the error $\left.\left\|u^{\omega, n}-u_{\omega, n}\right\|_{H^{k}}\right)$. Define $k:=s+2$. Then, for each $\omega \in\{-1,1\}$ and $n \geq n_{0}$ we have that

$$
u_{\omega, n} \in C\left(\left[0, T_{u}\right], H^{k+1}(\mathbb{S})\right) \cap C^{1}\left(\left[0, T_{u}\right], H^{k}(\mathbb{S})\right) .
$$

Moreover, there is a constant $C\left(T_{u}\right)>0$ such that

$$
\max _{t \in\left[0, T_{u}\right]}\left\|u^{\omega, n}(t)-u_{\omega, n}(t)\right\|_{H^{k}} \leq C\left(T_{u}\right) n^{2} \quad \text { for all } \omega \in\{-1,1\}, n \geq n_{0} .
$$

Proof. Let $\omega \in\{-1,1\}$ and let $n \geq n_{0}$ be arbitrary. For simplicity we set $u:=u_{\omega, n}$. Because $u(0)$ is smooth, we know in view of Theorem 2.1 that the Cauchy problem (18) has a unique maximal solution

$$
u \in C\left([0, T), H^{k+1}(\mathbb{S})\right) \cap C^{1}\left([0, T), H^{k}(\mathbb{S})\right)
$$

with existence time $T:=T(\omega, n)$. In order to derive a bound on the absolute error in $H^{k}$ we have to prove first that this additional regularity holds up to and including the time $T_{u}$. That is, we have to show that $T>T_{u}$ for all $\omega \in\{-1,1\}$ and $n \geq n_{0}$. To this end we proceed as in the proof of Theorem 2.1 and compute that

$$
\frac{d}{d t}\|u\|_{H^{k}}^{2} \leq C\left(\|u\|_{W_{\infty}^{1}}\|u\|_{H^{k}}^{2}+\|u\|_{H^{k}}\left\|\Lambda^{k-2} \partial_{x} R(u)\right\|_{L_{2}}\right) \quad \text { in }[0, T) .
$$


We study the last term more carefully and obtain in view of the commutator estimate (2) that

$$
\begin{aligned}
\left\|\Lambda^{k-2} \partial_{x}\left(u_{x}^{2}\right)\right\|_{L_{2}} & \leq 2\left\|\left[\Lambda^{k-2}, u_{x}\right] u_{x x}\right\|_{L_{2}}+2\left\|u_{x} \Lambda^{k-2} u_{x x}\right\|_{L_{2}} \\
& \leq C\left(\|u\|_{W_{\infty}^{2}}\|u\|_{H^{k-1}}+\|u\|_{W_{\infty}^{1}}\|u\|_{H^{k}}\right)
\end{aligned}
$$

whereas

$$
\left\|\Lambda^{k-2} \partial_{x} u\right\|_{L_{2}} \leq C\|u\|_{H^{k-1}}
$$

and

$$
\begin{aligned}
\left\|\Lambda^{k-2} \partial_{x}\left(u^{p}\right)\right\|_{L_{2}} & \leq p\left\|\left[\Lambda^{k-2}, u^{p-1}\right] u_{x}\right\|_{L_{2}}+p\left\|u^{p-1} \Lambda^{k-2} u_{x}\right\|_{L_{2}} \\
& \leq C\left(\|u\|_{W_{\infty}^{1}}^{p-1}\|u\|_{H^{s}}+\|u\|_{W_{\infty}^{1}}\|u\|_{H^{s}}^{p-1}+\|u\|_{L_{\infty}}^{p-1}\|u\|_{H^{k-1}}\right)
\end{aligned}
$$

for $2 \leq p \leq 4$. In view of the embedding $H^{s}(\mathbb{S}) \hookrightarrow C^{1}(\mathbb{S})$ and (19) we find that

$$
\frac{d}{d t}\|u\|_{H^{k}}^{2} \leq C\left(\|u\|_{H^{k}}^{2}+\|u\|_{W_{\infty}^{2}}\|u\|_{H^{k-1}}\|u\|_{H^{k}}\right) \quad \text { in }\left[0, \min \left\{T, T_{u}\right\}\right) .
$$

Next we employ the well-known interpolation inequality

$$
\|u\|_{H^{r}} \leq\|u\|_{H^{r_{1}}}^{\left(r_{2}-r\right) /\left(r_{2}-r_{1}\right)}\|u\|_{H^{r_{2}}}^{\left(r-r_{1}\right) /\left(r_{2}-r_{1}\right)}
$$

for $r=k-1, r_{1}=s$ and $r_{2}=k$ and obtain that $\|u\|_{H^{k-1}}^{2} \leq\|u\|_{H^{s}}\|u\|_{H^{k}}$ for all $u \in H^{k}(\mathbb{S})$. Recalling that $H^{k-1}(\mathbb{S}) \hookrightarrow C^{2}(\mathbb{S})$, we arrive at

$$
\frac{d}{d t}\|u\|_{H^{k}}^{2} \leq C\|u\|_{H^{k}}^{2} \quad \text { in }\left[0, \min \left\{T, T_{u}\right\}\right)
$$

which we may integrate with respect to time to obtain

$$
\|u\|_{H^{k}} \leq e^{C T_{u}}\|u(0)\|_{H^{k}} \quad \text { in }\left[0, \min \left\{T, T_{u}\right\}\right) .
$$

This inequality shows that $T>T_{u}$ for $\omega \in\{-1,1\}$ and for all $n \geq n_{0}$. Indeed, assuming to the contrary that $T<T_{u}$, then $\|u(t)\|_{H^{k}} \rightarrow \infty$ as $t$ approaches the maximal existence time $T$ of $u \in H^{k}$. This is a contradiction to the fact that $u$ is bounded in $H^{k}$ in view of (22). Finally, the error estimate (20) is a simple consequence of (22) and of the estimate

$$
\|u(0)\|_{H^{k}}=\left\|u^{\omega, n}(0)\right\|_{H^{k}} \leq C n^{k-s}
$$

for all $n \geq n_{0}$, cf. (13).

It turns out that estimate (20) can be improved when we choose $k=1$ and $s \geq 2$. The argument relies on the regularity properties derived in the previous Lemma 4.1.

Lemma 4.2 (Estimating the error $\left\|u^{\omega, n}-u_{\omega, n}\right\|_{H^{1}}$ ). Assume that $s \geq 2$. Then, for all $n \geq n_{0}$ and $\omega \in\{-1,1\}$ we have that

$$
\max _{t \in\left[0, T_{u}\right]}\left\|u^{\omega, n}(t)-u_{\omega, n}(t)\right\|_{H^{1}} \leq C\left(T_{u}\right) n^{-s} .
$$

Proof. Denoting the difference between the approximate solution and the exact solution by $v:=u^{\omega, n}-u_{\omega, n}$, we see that $v$ is a solution of the initial value problem

$$
\left\{\begin{array}{rll}
v_{t} & =v_{x}-14 v v_{x}+14 u^{\omega, n} v_{x}+14 u_{x}^{\omega, n} v+E+\partial_{x} \Lambda^{-2}(F) & \text { for } t>0, \\
v(0) & =0,
\end{array}\right.
$$


whereby $E$ is the error term defined by (14) and

$$
\begin{aligned}
F:= & R\left(u^{\omega, n}\right)-R\left(u_{\omega, n}\right) \\
= & 14 u_{x}^{\omega, n} v_{x}-7 v_{x}^{2}-2 v-20 u^{\omega, n} v+10 v^{2}+6\left(u^{\omega, n}\right)^{2} v-6 u^{\omega, n} v^{2}+2 v^{3} \\
& -12\left(u^{\omega, n}\right)^{3} v+18\left(u^{\omega, n}\right)^{2} v^{2}-12 u^{\omega, n} v^{3}+3 v^{4} .
\end{aligned}
$$

In view of the regularity property derived for $u_{\omega, n}$ in Lemma 4.1 , we may apply $\Lambda^{2}$ on both sides of (24) and find that

$$
\Lambda^{2} v_{t}=\Lambda^{2} v_{x}-14 \Lambda^{2}\left(v v_{x}\right)+14 \Lambda^{2}\left(u^{\omega, n} v_{x}\right)+14 \Lambda^{2}\left(u_{x}^{\omega, n} v\right)+\Lambda^{2} E+\partial_{x} F
$$

and therewith

$$
\begin{aligned}
\frac{1}{2} \frac{d}{d t}\|v\|_{H^{1}}^{2}= & \int_{\mathbb{S}} v \Lambda^{2} v_{t} d x \\
= & \int_{\mathbb{S}} v \Lambda^{2} v_{x} d x-14 \int_{\mathbb{S}} v \Lambda^{2}\left(v v_{x}\right) d x+14 \int_{\mathbb{S}} v \Lambda^{2}\left(u^{\omega, n} v_{x}\right) d x \\
& +14 \int_{\mathbb{S}} v \Lambda^{2}\left(u_{x}^{\omega, n} v\right) d x+\int_{\mathbb{S}} v \Lambda^{2} E d x+\int_{\mathbb{S}} v \partial_{x} F d x
\end{aligned}
$$

for all $t \in\left[0, T_{u}\right]$. Taking into account that

$$
\int_{\mathbb{S}} v \Lambda^{2} v_{x} d x=\int_{\mathbb{S}} v v_{x}+v_{x} v_{x x} d x=0
$$

and noting that

$$
14 \int_{\mathbb{S}} v \Lambda^{2}\left(v v_{x}\right) d x=14 \int_{\mathbb{S}} v^{2} v_{x} d x+7 \int_{\mathbb{S}} v_{x}^{3} d x=7 \int_{\mathbb{S}} v_{x}^{3} d x
$$

we find

$$
\begin{aligned}
\frac{d}{d t}\|v\|_{H^{1}}^{2}= & -7 \int_{\mathbb{S}} v_{x}^{3} d x+14 \int_{\mathbb{S}} v \Lambda^{2}\left(u^{\omega, n} v_{x}\right) d x+14 \int_{\mathbb{S}} v \Lambda^{2}\left(u_{x}^{\omega, n} v\right) d x+\int_{\mathbb{S}} v \Lambda^{2} E d x \\
& -\int_{\mathbb{S}} v_{x}\left(14 u_{x}^{\omega, n} v_{x}-7 v_{x}^{2}-2 v-20 u^{\omega, n} v+10 v^{2}+6\left(u^{\omega, n}\right)^{2} v-6 u^{\omega, n} v^{2}\right) d x \\
& -\int_{\mathbb{S}} v_{x}\left(2 v^{3}-12\left(u^{\omega, n}\right)^{3} v+18\left(u^{\omega, n}\right)^{2} v^{2}-12 u^{\omega, n} v^{3}+3 v^{4}\right) d x \\
= & 14 \int_{\mathbb{S}} v \Lambda^{2}\left(u^{\omega, n} v_{x}\right) d x+14 \int_{\mathbb{S}} v \Lambda^{2}\left(u_{x}^{\omega, n} v\right) d x+\int_{\mathbb{S}} v \Lambda^{2} E d x \\
& -\int_{\mathbb{S}} v_{x}\left(14 u_{x}^{\omega, n} v_{x}-20 u^{\omega, n} v+6\left(u^{\omega, n}\right)^{2} v-6 u^{\omega, n} v^{2}\right) d x \\
& -\int_{\mathbb{S}} v_{x}\left(-12\left(u^{\omega, n}\right)^{3} v+18\left(u^{\omega, n}\right)^{2} v^{2}-12 u^{\omega, n} v^{3}\right) d x .
\end{aligned}
$$

This leads us to the following inequality

$$
\begin{gathered}
\frac{d}{d t}\|v(t)\|_{H^{1}}^{2} \leq C \\
+\left\|u_{x}^{\omega, n}\right\|_{W_{\infty}^{1}}\|v\|_{H^{1}}^{2}+\|E\|_{H^{1}}\|v\|_{H^{1}}+\left(1+\left\|u^{\omega, n}\right\|_{L_{\infty}}\right)^{2}\left\|u_{x}^{\omega, n}\right\|_{L_{\infty}}\|v\|_{H^{1}}^{2} \\
\left.+\left\|u^{\omega, n}\right\|_{L_{\infty}}\left\|u_{x}^{\omega, n}\right\|_{L_{\infty}}\|v\|_{H^{1}}^{3}+\left\|u_{x}^{\omega, n}\right\|_{L_{\infty}}\|v\|_{H^{1}}^{4}\right) .
\end{gathered}
$$

Observing that the relation (13) implies $\sup _{\left[0, T_{u}\right]}\left\|u^{\omega, n}(t)\right\|_{H^{2}} \leq C$, we find together with (19) that

$$
\frac{d}{d t}\|v\|_{H^{1}}^{2} \leq C\left(\left\|u_{x}^{\omega, n}\right\|_{W_{\infty}^{1}}\|v\|_{H^{1}}^{2}+\|E\|_{H^{1}}\|v\|_{H^{1}}+\left\|u_{x}^{\omega, n}\right\|_{L_{\infty}}\|v\|_{H^{1}}^{2}\right)
$$


Taking now into account the estimates

$$
\left\|u_{x}^{\omega, n}\right\|_{L_{\infty}} \leq C n^{1-s} \quad \text { and } \quad\left\|u_{x}^{\omega, n}\right\|_{W_{\infty}^{1}} \leq C n^{2-s}
$$

for $n \geq n_{0}$ and $\omega \in\{-1,1\}$, we obtain in view of the error estimate (15) in Lemma 3.1 that

$$
\frac{d}{d t}\|v\|_{H^{1}}^{2} \leq C\left(\|v\|_{H^{1}}^{2}+n^{-s}\|v\|_{H^{1}}\right) .
$$

The latter estimate leads us to

$$
\frac{d}{d t}\|v\|_{H^{1}} \leq C\left(\|v\|_{H^{1}}+n^{-s}\right) \quad \text { in }\left[0, T_{u}\right],
$$

the desired estimate (23) following in view of Gronwall's inequality and since $v(0)=0$.

Before proving the main result, we show the analog of Lemma 4.2 in the situation when $3 / 2<s<2$. The regularity properties derived in Lemma 4.1 are once again essential.

Lemma 4.3 (Estimating the error $\left\|u^{\omega, n}-u_{\omega, n}\right\|_{H^{\sigma}}$ ). Let $3 / 2<s<2$. For all $n \geq n_{0}$, $\omega \in\{-1,1\}$ and $1 / 2<\sigma \leq s-1$ we have that

$$
\max _{t \in\left[0, T_{u}\right]}\left\|u^{\omega, n}(t)-u_{\omega, n}(t)\right\|_{H^{\sigma}} \leq C\left(T_{u}\right) n^{-s} .
$$

Proof. In this case, we interpret the function $v=u^{\omega, n}-u_{\omega, n}$ as a solution of the initial value problem

$$
\left\{\begin{aligned}
v_{t} & =v_{x}+7\left(\left(u^{\omega, n}+u_{\omega, n}\right) v\right)_{x}+E+\partial_{x} \Lambda^{-2} G \quad \text { for } t>0 \\
v(0) & =0
\end{aligned}\right.
$$

with $E$ given by (14) and with $G:=R\left(u^{\omega, n}\right)-R\left(u_{\omega, n}\right)$. It is useful to bring $G$ in the following form

$$
\begin{aligned}
G= & 7\left(u^{\omega, n}+u_{\omega, n}\right)_{x} v_{x}-3 v\left(\left(u^{\omega, n}\right)^{3}+\left(u^{\omega, n}\right)^{2} u_{\omega, n}+u^{\omega, n}\left(u_{\omega, n}\right)^{2}+\left(u_{\omega, n}\right)^{3}\right) \\
& +2 v\left(\left(u^{\omega, n}\right)^{2}+u^{\omega, n} u_{\omega, n}+\left(u_{\omega, n}\right)^{2}\right)-10 v\left(u^{\omega, n}+u_{\omega, n}\right)-2 v
\end{aligned}
$$

In view of (26), we have

$$
\begin{aligned}
\frac{1}{2} \frac{d}{d t}\|v\|_{H^{\sigma}}^{2}=\int_{\mathbb{S}} \Lambda^{\sigma} v \Lambda^{\sigma} v_{t} d x= & \int_{\mathbb{S}} \Lambda^{\sigma} v \Lambda^{\sigma} v_{x} d x+\int_{\mathbb{S}} \Lambda^{\sigma} v \Lambda^{\sigma} E d x \\
& +7 \int_{\mathbb{S}} \Lambda^{\sigma} v \Lambda^{\sigma}\left(\left(u^{\omega, n}+u_{\omega, n}\right) v\right)_{x} d x+\int_{\mathbb{S}} \Lambda^{\sigma} v \Lambda^{\sigma} \partial_{x} \Lambda^{-2} G d x
\end{aligned}
$$

The first term in the previous equation vanishes

$$
\int_{\mathbb{S}} \Lambda^{\sigma} v \Lambda^{\sigma} v_{x} d x=0
$$

while applying the Cauchy-Schwarz inequality for the second and fourth term we obtain the estimates

$$
\begin{aligned}
\left\|\Lambda^{\sigma} v \Lambda^{\sigma} E\right\|_{L_{1}} & \leq\|v\|_{H^{\sigma}}\|E\|_{H^{\sigma}} \\
\left\|\Lambda^{\sigma} v \Lambda^{\sigma} \partial_{x} \Lambda^{-2} G\right\|_{L_{1}} & \leq\|v\|_{H^{\sigma}}\|G\|_{H^{\sigma-1}} .
\end{aligned}
$$

To derive a bound for the third term, we use the Calderon-Coifman-Meyer type estimate (3). We first commute the operator $\Lambda^{\sigma} \partial_{x}$ with the function $u^{\omega, n}+u_{\omega, n}$ and obtain

$$
\begin{aligned}
\int_{\mathbb{S}} \Lambda^{\sigma} v \Lambda^{\sigma}\left(\left(u^{\omega, n}+u_{\omega, n}\right) v\right)_{x} d x= & \int_{\mathbb{S}} \Lambda^{\sigma} v\left(u^{\omega, n}+u_{\omega, n}\right) \Lambda^{\sigma} \partial_{x} v d x \\
& +\int_{\mathbb{S}} \Lambda^{\sigma} v\left[\Lambda^{\sigma} \partial_{x},\left(u^{\omega, n}+u_{\omega, n}\right)\right] v d x .
\end{aligned}
$$


After integrating by parts, we estimate the first integral as follows

$$
\left|\int_{\mathbb{S}} \Lambda^{\sigma} v\left(u^{\omega, n}+u_{\omega, n}\right) \Lambda^{\sigma} \partial_{x} v d x\right| \leq\left\|\partial_{x}\left(u^{\omega, n}+u_{\omega, n}\right)\right\|_{L^{\infty}}\|v\|_{H^{\sigma}}^{2} .
$$

To estimate the second integral, we apply the Cauchy-Schwarz inequality and then use the estimate (3) to find

$$
\begin{aligned}
\left|\int_{\mathbb{S}} \Lambda^{\sigma} v\left[\Lambda^{\sigma} \partial_{x},\left(u^{\omega, n}+u_{\omega, n}\right)\right] v d x\right| & \leq\left\|\Lambda^{\sigma} v\right\|_{L^{2}}\left\|\left[\Lambda^{\sigma} \partial_{x}, u^{\omega, n}+u_{\omega, n}\right] v\right\|_{L_{2}} \\
& \leq C\left\|u^{\omega, n}+u_{\omega, n}\right\|_{H^{s}}\|v\|_{H^{\sigma}}^{2}
\end{aligned}
$$

In view of the boundedness of the family $\left\{\max _{\left[0, T_{u}\right]}\left\|u^{\omega, n}+u_{\omega, n}\right\|_{H^{s}}: n \geq n_{0}, \omega= \pm 1\right\}$ we may combine the preceding estimates and obtain that

$$
\left\|\Lambda^{\sigma} v \Lambda^{\sigma}\left(\left(u^{\omega, n}+u_{\omega, n}\right) v\right)_{x}\right\|_{L_{1}} \leq C\|v\|_{H^{\sigma}}^{2} .
$$

The latter argument and the multiplier inequality (4) show that

$$
\|G\|_{H^{\sigma-1}} \leq C\|v\|_{H^{\sigma}},
$$

and together with the error bound (15) obtained in Lemma 3.1 we conclude that

$$
\frac{d}{d t}\|v\|_{H^{\sigma}}^{2} \leq C\left(\|v\|_{H^{\sigma}}^{2}+n^{-2 s+1+\sigma}\|v\|_{H^{\sigma}}\right) .
$$

Whence,

$$
\frac{d}{d t}\|v\|_{H^{\sigma}} \leq C\left(\|v\|_{H^{\sigma}}+n^{-2 s+1+\sigma}\right) \quad \text { in }\left[0, T_{u}\right],
$$

and the conclusion follows, as in Lemma 4.2, by taking into account that $-2 s+1+\sigma \leq-s$ for all $\sigma \in(1 / 2, s-1]$.

\section{Proof of the main Result}

In the remaining part we prove that the functions $u_{n}:=u_{1, n+n_{0}}$ and $\widetilde{u}_{n}:=u_{-1, n+n_{0}}$, $n \in \mathbb{N}$, satisfy all the properties required in Theorem 1.1. Recalling the estimate (19), which ensures that the strong solutions $u_{ \pm 1, n}, n \geq n_{0}$, are bounded in $H^{s}$, proves the first claim

$$
\sup _{n \geq n_{0}} \max _{t \in\left[0, T_{u}\right]}\left\|u_{1, n}(t)\right\|_{H^{s}}+\left\|u_{-1, n}(t)\right\|_{H^{s}} \leq C,
$$

where $T_{u}$ is the constant introduced right before Lemma 4.1. The second assertion follows by taking into account the definition of the approximate solutions (11), which yields

$$
\left\|u_{1, n}(0)-u_{-1, n}(0)\right\|_{H^{s}}=\frac{2 n^{-1}}{14}\|1\|_{H^{s}} \rightarrow_{n \rightarrow \infty} 0 .
$$

To show that the third claim of Theorem 1.1 holds, we have to derive a decay estimate for the difference between the two unknown exact solutions. The trick is to work with inequalities involving the estimates for the absolute errors deduced in the preceding lemmas. We assume first that $s \geq 2$, and observe that

$$
\begin{aligned}
\left\|u_{1, n}(t)-u_{-1, n}(t)\right\|_{H^{s}} \geq & \left\|u^{1, n}(t)-u^{-1, n}(t)\right\|_{H^{s}} \\
& -\left\|u^{1, n}(t)-u_{1, n}(t)\right\|_{H^{s}}-\left\|u^{-1, n}(t)-u_{-1, n}(t)\right\|_{H^{s}}
\end{aligned}
$$


for all $t \in\left[0, T_{u}\right]$ and $n \geq n_{0}$. Now we find lower bounds for each of these three terms. A simple calculation yields that

$$
\begin{aligned}
\left\|u^{1, n}(t)-u^{-1, n}(t)\right\|_{H^{s}} & =\frac{1}{14}\left\|2 n^{-1}-n^{-s}(\cos (n x+t)-\cos (n x-t))\right\|_{H^{s}} \\
& \geq \frac{n^{-s}}{7}|\sin (t)|\|\sin (n x)\|_{H^{s}}-\frac{\sqrt{2 \pi} n^{-1}}{7} \\
& \geq \frac{\sqrt{\pi}|\sin (t)|}{7}-\frac{\sqrt{2 \pi} n^{-1}}{7}
\end{aligned}
$$

for all $n \geq n_{0}$ and $t \in\left[0, T_{u}\right]$, where we have used (12) in the last inequality. To estimate the second and third term in (27), we apply the interpolation inequality (21) with $r=s$, $r_{1}=1$ and $r_{2}=k$, and find

$$
\begin{aligned}
\left\|u^{ \pm 1, n}(t)-u_{ \pm 1, n}(t)\right\|_{H^{s}} & \leq\left\|u^{ \pm 1, n}(t)-u_{ \pm 1, n}(t)\right\|_{H^{1}}^{\frac{2}{k-1}}\left\|u^{ \pm 1, n}(t)-u_{ \pm 1, n}(t)\right\|_{H^{k}}^{\frac{s-1}{k-1}} \\
& \leq C\left(T_{u}\right) n^{\frac{-2}{k-1}}
\end{aligned}
$$

for all $n \geq n_{0}$, in view of the estimates (23) and (25) obtained in Lemma 4.1 and 4.2, respectively. Gathering (28) and (29), we obtain that

$$
\left\|u_{1, n}(t)-u_{-1, n}(t)\right\|_{H^{s}} \geq \frac{\sqrt{\pi}|\sin (t)|}{7}-\frac{\sqrt{2 \pi} n^{-1}}{7}-C\left(T_{u}\right) n^{\frac{-2}{k-1}},
$$

for all $t \in\left[0, T_{u}\right]$ and $n \geq n_{0}$. Finally, we let $n \rightarrow \infty$ to complete the proof in the case $s \geq 2$.

For $3 / 2<s<2$ we note that the estimate (28) is still valid, whereas the analog of (29) holds in view of Lemma 4.3. Indeed, we find that

$$
\begin{aligned}
\left\|u^{ \pm 1, n}(t)-u_{ \pm 1, n}(t)\right\|_{H^{s}} & \leq\left\|u^{ \pm 1, n}(t)-u_{ \pm 1, n}(t)\right\|_{H^{\sigma}}^{\frac{2}{k-\sigma}}\left\|u^{ \pm 1, n}(t)-u_{ \pm 1, n}(t)\right\|_{H^{k}}^{\frac{s-\sigma}{k-\sigma}} \\
& \leq C\left(T_{u}\right) n^{\frac{-2 \sigma}{k-\sigma}},
\end{aligned}
$$

where $\sigma \in(1 / 2, s-1]$ is fixed and $n \geq n_{0}$. The final argument of the proof is analogous to the one presented in the case when $s \geq 2$.

Acknowledgements. A. Geyer was supported by the FWF project J 3452 "Dynamical Systems Methods in Hydrodynamics" of the Austrian Science Fund.

\section{REFERENCES}

[1] A. Boutet de Monvel, A. Kostenko, D. Shepelsky, and G. Teschl. Long-time asymptotics for the Camassa-Holm equation. SIAM J. Math. Anal., 41(4):1559-1588, 2009.

[2] A. Bressan and A. Constantin. Global conservative solutions of the Camassa-Holm equation. Arch. Ration. Mech. Anal., 183(2):215-239, 2007.

[3] R. Camassa and D. D. Holm. An integrable shallow water equation with peaked solitons. Phys. Rev. Lett., 71(11):1661-1664, 1993.

[4] A. Constantin. On the scattering problem for the Camassa-Holm equation. R. Soc. Lond. Proc. Ser. A Math. Phys. Eng. Sci., 457(2008):953-970, 2001.

[5] A. Constantin. Nonlinear Water Waves with Applications to Wave-Current Interactions and Tsunamis, volume 81 of CBMS-NSF Conference Series in Applied Mathematics. SIAM, Philadelphia, 2011.

[6] A. Constantin and J. Escher. Well-posedness, global existence, and blowup phenomena for a periodic quasi-linear hyperbolic equation. Comm. Pure Appl. Math., 51(5):475-504, 1998.

[7] A. Constantin, V. S. Gerdjikov, and R. I. Ivanov. Inverse scattering transform for the Camassa-Holm equation. Inverse Problems, 22(6):2197-2207, 2006.

[8] A. Constantin and D. Lannes. The hydrodynamical relevance of the Camassa-Holm and DegasperisProcesi equations. Arch. Ration. Mech. Anal., 192(1):165-186, 2009. 
[9] N. Duruk Mutlubaş and A. Geyer. Orbital stability of solitary waves of moderate amplitude in shallow water. J. Differential Equations, 255(2):254-263, 2013.

[10] N. Duruk Mutlubaş. On the Cauchy problem for a model equation for shallow water waves of moderate amplitude. Nonlinear Anal. Real World Appl., 14(5):2022-2026, 2013.

[11] N. Duruk Mutlubaş. Local well-posedness and wave breaking results for periodic solutions of a shallow water equation for waves of moderate amplitude. Nonlinear Anal., 97:145-154, 2014.

[12] J. Escher and B. Kolev. Geodesic completness for Sobolev $H^{s}$-metrics on the diffeomorphisms group of the circle. 2013. arXiv:1308.357v1.

[13] Y. Fu and Z. Liu. Non-uniform dependence on initial data for the periodic modified Camassa-Holm equation. NoDEA Nonlinear Differential Equations Appl., 20:741-755, 2013.

[14] A. Geyer. Solitary traveling waves of moderate amplitude. J. Nonlinear Math. Phys., 19(supp01):12p, 2012.

[15] M. Grillakis, J. Shatah, and W. Strauss. Stability theory of solitary waves in the presence of symmetry. J. Funct. Anal., 74:160-197, 1987.

[16] A. A. Himonas and C. Kenig. Non-uniform dependence on initial data for the CH equation on the line. Differential Integral Equations, 22:201-224, 2009.

[17] A. A. Himonas, C. Kenig, and G. Misiolek. Non-uniform dependence for the periodic CH equation. Commun. Partial Differential Equations, 35:1145-1162, 2010.

[18] A. A. Himonas and G. Misiolek. Non-uniform dependence on initial data of solutions to the Euler Equations of hydrodynamics. Comm. Math. Phys, 296(1):285-301, 2010.

[19] R. S. Johnson. Camassa-Holm, Korteweg-de Vries and related models for water waves. J. Fluid Mech., 455:63-82, 2002.

[20] D. Karapetyan. Non-uniform dependence and well-posedness for the hyperelastic rod equation. J. Differential Equations, 249:796-826, 2010.

[21] T. Kato. Quasi-linear equations of evolution, with applications to partial differential equations. In Spectral theory and differential equations (Proc. Sympos., Dundee, 1974; dedicated to Konrad Jörgens), pages 25-70. Lecture Notes in Math., Vol. 448. Springer, Berlin, 1975.

[22] T. Kato and G. Ponce. Commutator estimates and the euler and Navier-Stokes equations. Comm. Pure Appl. Math., 41(7):891-907, 1988.

[23] G. Lv, P. Y. H. Pang, and M. Wang. Non-uniform dependence on initial data for the $\mu-b$ equation. Z. Angew. Math. Phys, 64(5):1543-1554, 2013.

[24] G. Lv and M. Wang. Non-uniform dependence for a modified Camassa-Holm system. J. Math. Physics, 53:013101, 2012.

[25] H. P. McKean. Breakdown of a shallow water equation. Asian J. Math., 2(4):867-874, 1998.

[26] Y. Mi and C. Mu. On the solutions of a model equation for shallow water waves of moderate amplitude. J. Differential Equations, 255:2101-2129, 2013.

[27] G. Misiolek. Classical Solutions of the periodic Camassa-Holm equation. Geom. Funct. Anal., 12(5):1080-1104, 2002.

[28] T. Runstl and W. Sickel. Sobolev Spaces of Fractional Order, Nemytskij Operators, and Nonlinear Partial Differential Equations, volume 35 of de Gruyter Series in Nonlinear Analysis and Applications. Walter de Gruyter \& Co, Berlin, 1996.

[29] M. Taylor. Pseudodifferential Operators and Nonlinear PDE. Birkhäuser, Boston, 1991.

[30] M. Taylor. Commutator estimates. Proc. Amer. Math. Soc., 131:1501-1507, 2002.

Istanbul Kemerburgaz University, School of Arts and Sciences, Department of Basic Sciences, Mahmutbey Dilmenler Caddesi, No:26, 34217 BaĞcilar, Istanbul, Turkey.

E-mail address: nilay.duruk@kemerburgaz.edu.tr

Departament de Matemàtiques, Universitat Autònoma de Barcelona, Facultat de Ciències, 08193 Bellaterra, Barcelona, Spain.

E-mail address: annageyer@mat.uab.cat

Institut für Angewandte Mathematik, Leibniz Universität Hannover, Welfengarten 1, 30167 Hannover, Germany.

E-mail address: matioc@ifam.uni-hannover.de 